\title{
Universality of evolutionary dynamics with arbitrary demography
}

\author{
Andrea Mazzolini ${ }^{1,2}$ and Jacopo Grilli ${ }^{2}$, \\ ${ }^{1}$ Laboratoire de physique de lcole normale suprieure (PSL University), \\ CNRS, Sorbonne Universit, and Universit de Paris, 75005 Paris, France \\ ${ }^{2}$ The Abdus Salam International Centre for Theoretical Physics (ICTP), Strada Costiera 11, 34014 Trieste, Italy
}

The assumption of constant population size is central in population genetics. It led to a large body of results, that are robust to modeling choices and that have proven successful to understand evolutionary dynamics. In reality, allele frequencies and population size are both determined by the interaction between a population and the environment. Including explicitly the demographic factors and life-history traits that determine the eco-evolutionary dynamics makes the analysis difficult and the results contingent on model details. Here, we develop a framework that encompasses a great variety of systems with arbitrary population dynamics and competition between species. By using techniques based on scale separation for stochastic processes, we are able to calculate analytically evolutionary properties, such as the fixation probability. Remarkably, these properties assume a universal form with respect to our framework, which depends on only three life-history traits related to the inter-generation timescale, the invasion fitness, and the carrying capacity of the alleles. In other words, different systems, such as Lotka-Volterra or a chemostat model, share the same evolutionary outcomes after mapping the parameters of the models into three effective life-history traits. An important and surprising consequence of our results is that the direction of selection can be inverted, with a population evolving to reach lower values of invasion fitness.

Competition is a fundamental agent of natural selection. The scarcity of a resource (a nutrient, water, space, ...) limits the population growth, determining a selection pressure. Variants that are able to grow faster or more efficiently spread within the population. To quantify these kinds of phenomena, the vast majority of results in population genetics are based on the assumption of constant population size [1] 8 . The success of this assumption lies in the generality of the results. When the population size is large enough, Kimura's diffusion limit [1, 9] is the convergence point of several alternative models. In fact, while different population genetics models (Wrigh-Fisher [10, 11], Moran [12, conditional branching processes [13, and some Canning processes [14]) start from radically different assumptions about the genealogical and demographic structure of the population, they share the same predictions, up to a simple rescaling of timescales and parameters. For most of the theoretical advances in population genetics, the total population size is treated as an effective parameter of the model, which should be fitted from data, and the allele frequencies are the only dynamical degrees of freedom.

However, mechanistically, not only the allele frequencies but also the total population size is determined by the interaction between a population and the environment. Moreover, experimental works [15, 16] suggest that the variation of the population size can play a role in the evolutionary process, implying that its dynamics should not be neglected in theoretical descriptions.

Non-stationary conditions are an important example where the dynamics of population size cannot be neglected to understand how evolution - here intended as the dynamics of relative frequencies of alleles - unfolds. For instance, range expansions leave strong signature on the genetic diversity of a population [17-19].

\footnotetext{
*Electronic address: jgrilli@ictp.it
} 
A classical approach to study evolution under stationary, but not constant, total population size is to consider the dynamics of the latter as decoupled from the allele frequency, by assuming a-priori the dynamics of the total population $20-23]$.

Here we instead focus on the equally non-trivial scenarios where the total population admits a stationary distribution, but its fluctuations are not independent of allele frequencies, over the timescales of population dynamics. This approach requires to model how environmental constraints limit population growth, for instance by considering Lotka-Volterra dynamics 24-30] or generalizations of the Moran model [31.

The increased realism of these models undermine, at least in principle, the generality of the standard population genetics results. To what extent do the details of demography and life-history determine the evolutionary outcome? For instance, will a population whose growth is described by a logistic model differ in its evolutionary trajectory from a population described by Gompertz growth?

In this work, we address this question by considering a general ecological framework to describe population dynamics. This approach encompasses several alternative models, from Lotka-Volterra to competition in a chemostat, under the same mathematical framework. We show that evolutionary predictions are instead robust and insensitive to the details of population dynamics. Within our framework, evolutionary observables, such as the fixation probability, are universal and depend on only three parameters, related to the concepts of inter-generation timescale, invasion fitness, and carrying capacity. We first present the general framework, then we show its approximation with the scale separation and the computation of evolutionary observables. Finally, we conclude with an explicit example of the chemostat model for resource competition, showing how the previous general results can lead to an evolutionary trajectory with decreasing fitness over time.

Results

\section{A model for evolution with a general demographic dynamics}

The growth curve of a clonal population displays a typical sigmoid shape (see Fig. 1): a rapid initial growth followed by a deceleration due to nutrient limitation, and, eventually, a convergence of the abundance to a carrying capacity, determined by the availability and quality of nutrients. Several different models can be used to describe the growth of a population under limited nutrients. These models differ in the specific functional form of the population growth trajectory.

Two essential parameters capture the dimensional components of these growth curves. The carrying capacity K defines the values of population abundance reached at large times. A timescale $T$ sets the speed of convergence of population curves to carrying capacity. Fig. 1 1 a shows the example of two alleles, each characterized by different values of $K$ and $T$, under different growth scenarios, specified by different commonly studied models.

While $K$ and $T$ are enough to specify the scales of the growth curves obtained in isolation, they are generally not enough to describe the dynamics in co-cultures. In particular, we are typically interested in describing pairwise competition experiments, where a single copy (or a small population) of a mutant allele is inoculated in a large population of a resident allele. In absence of frequency-dependent selection or of multiple substitutable resources, the outcomes of invasion experiments can be predicted by a single, allele-specific, number $\phi$ which we will refer to as 
invasion fitness (see Fig. 1b). The growth rate of a mutant is proportional to the relative invasion fitness difference with the resident population.

The setting outlined in Fig. 1 is common to a large plethora of models, such as Logistic, Gompertz or Von Bertalanffy growth. While these models are not always expressed explicitly in terms of the three quantities $T, K$ and $\phi$, their parameters can be written in terms of them. To show this, we introduce a general modeling framework describing the dynamics of multiple alleles competing for a single resource, defined by the following equation

$$
\frac{1}{n_{i}} \frac{d n_{i}}{d t}=\frac{1}{T_{i}} \beta\left(\sum_{j} \frac{n_{j}}{K_{j}} u_{j}\right)\left(1-\frac{1}{\phi_{i}} \omega\left(\sum_{j} \frac{n_{j}}{K_{j}} u_{j}\right)\right),
$$

where $u_{i}=\omega^{-1}\left(\phi_{i}\right)$. The positive functions $\beta(z)$ and the positive monotonically increasing function $\omega(z)$ (see Supplementary Materials, section S1) determine the specific shape of growth curves, which distinguish between alternative models.

While eq. 1 might appear cumbersome, it is easy to show that the growth curve has the same behavior depicted in Fig. 1 and the parameters $K, T$, and $\phi$ the same interpretation. Eq. 1 reduces in fact to standard models under specific choices of the two functions $\beta(z)$ and $\omega(z)$. Logistic growth is obtained using $\beta(z)=1$ and $\omega(z)=z$, the chemostat model [32] with $\beta(z)=1 / z$ and $\omega(z)=z$, Gompertz growth for $\beta(z)=1$ and $\omega(z)=\log (z)$, and Von Bertalanffy model for $\beta(z)=z^{\alpha-1}$ and $\omega(z)=z^{1-\alpha}$ (see Materials and Methods for more details and other examples). Eq. 1 is deterministic, and can be thought of as the approximation — or more precisely, the deterministic limit — of a stochastic process describing the stochastic dynamics of a heterogeneous population. We consider a birth-death process that generalizes the classic Moran model and reduces to eq. 1 in the deterministic limit (see Materials and Methods). In this model, contrarily to standard models in population genetics, both the abundances of each allele $n_{i}$ and the total population size $N=\sum_{i} n_{i}$ are changing stochastically over time in an interdependent manner. The population size $N$ is therefore not fixed but oscillates around a typical value, determined by the carrying capacities $K_{i}$ and the invasion fitnesses $\phi_{i}$.

We are interested in the limit of large population sizes, which in our context corresponds to $K_{i} \gg 1$. In this limit, classic models of population genetics (such as the Moran model or Wright-Fisher model) converge to the same effective diffusive limit, which corresponds to Kimura's equation.

\section{The effective diffusive dynamics is controlled by few parameters}

After a calculation based on scale separation [33] (see Materials and Methods and Supplementary Materials section S2) we obtain an effective equation, which parallels Kimura's diffusion limit. The diffusion limit, as in standard population genetics, holds for small fitness differences $\left(\left|\phi_{i}-\phi_{j}\right| / \phi_{i} \ll 1\right)$ and large population sizes $\left(K_{i} \gg 1\right)$.

In the case of two alleles, this effective equation describes the dynamics of the relative abundance $x=n_{1} /\left(n_{1}+n_{2}\right)$ of one allele, characterized by some values of timescale $T_{1}$, invasion fitness $\phi_{1}$, and carrying capacity $K_{1}$ in the presence of another allele with different traits $\left(T_{2}, K_{2}, \phi_{2}\right)$. The equation reads:

$$
\frac{d x}{d t}=\gamma_{r, c}(x) \frac{x(1-x)}{T_{1}}\left(s-\frac{\sigma_{r, c}(x)}{K_{2}}\right)+\sqrt{\frac{x(1-x)}{K_{2} T_{1}} \delta_{r, c}(x)} \cdot \eta(t) .
$$




\section{(a) Growth in isolation}
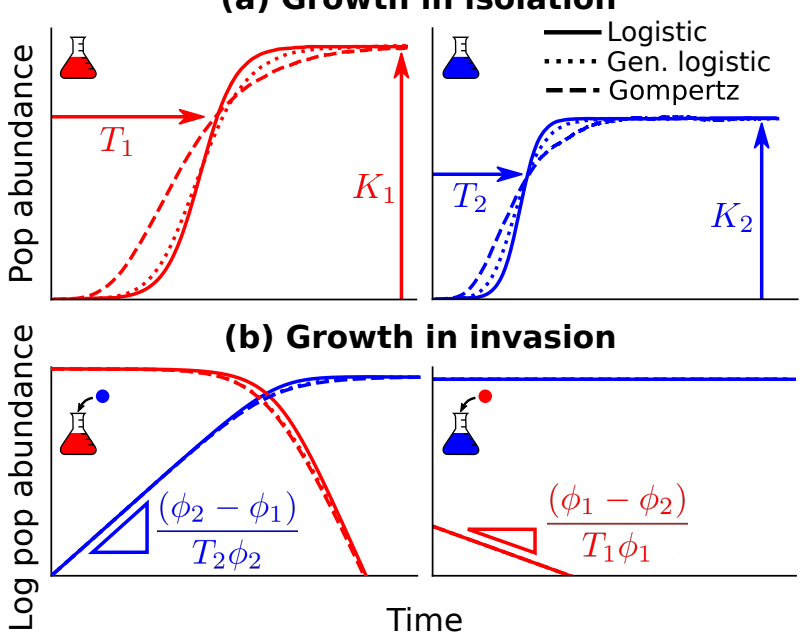

FIG. 1: The panel (a) shows a typical growth curve of a population in isolation, characterized by a carrying capacity $K_{i}$ and a timescale to reach the saturation $T_{i}$. In panel (b) one allele tries to invade tho other one at carrying capacity. The invasion success is determined by the invasion fitness $\phi_{i}$.

where $\eta(t)$ is a white noise term (see Supplementary Materials section S2). In addition to $K_{2}$ and $T_{1}$, the dynamics of the relative abundance $x$ depends on three parameters $(s, r, c)$. These three parameters are related to the ratios between the life-history trait parameters of the two alleles. In particular, $s$ is the selection coefficient $s=\left(\phi_{1}-\phi_{2}\right) / \phi_{1}$ while $r=T_{2} / T_{1}$, and $c=K_{2} / K_{1}$.

The trajectories of two populations growing together are therefore determined also by differences in inter-generation times, $r \neq 1$, and in carrying capacities, $c \neq 1$, through the functions $\gamma_{r, c}(x), \sigma_{r, c}(x)$ and $\delta_{r, c}(x)$ (see Materials and Methods for explicit definitions and Supplementary Materials for derivation). If the inter-generation times $T$ and the carrying capacities $K$ are equal (i.e., if $r=c=1$ ), eq. 2 reduces to Kimura's diffusion limit. In fact, $\gamma_{1,1}(x)=\delta_{1,1}(x)=1$ and $\sigma_{1,1}(x)=0$.

The deterministic term of equation 2 depends on the balance between two forces. The first term is proportional to the selection coefficient $s$. The second one, absent in the classic Kimura's diffusion limit, is proportional to $1 / K_{2}$ and depends also on allele frequencies, the time scales and the carrying capacities.

To better understand this point, it is instructive to consider the growth of the population of a rare allele with relative abundance $x$. If the allele is rare $(x \ll 1)$ the initial growth (or decline) will be approximately described by a stochastic exponential growth. In particular, the average relative abundance $\langle x\rangle$ is determined by (see Materials and Methods)

$$
\frac{d\langle x\rangle}{d t}=\frac{\langle x\rangle}{T_{1}}\left(s-\frac{(2 c-1)(r-1)}{K_{2}}\right)
$$

If the carrying capacity of the resident population is very large, $K_{2} \rightarrow \infty$, the growth rate converges to the deterministic limit $s / T_{1}$, depicted in Fig. 1 b. More generally, the deterministic limit is correct whenever $s K_{2} \gg(1-2 c)(1-r)$.

On the other hand, the presence of large but finite population sizes and large enough trait variation drastically change the relative abundance trajectories. Under some choices of $c$ and $r$, it can happen that an invader having smaller invasion fitness can grow on average within the "fitter" resident population. To better quantify this statement, in the next section we compare the fixation probabilities of the two alleles. 


\section{(a) Log-invasion-prob.ratio $=0$ surface}

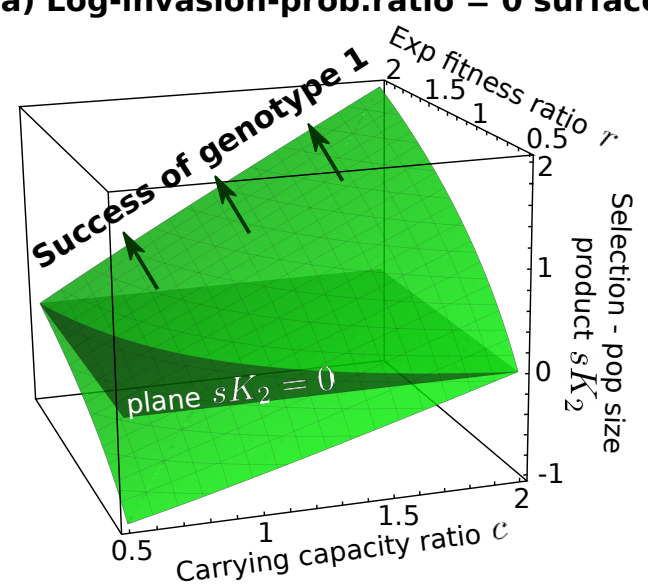

(b) Sections of the log-invasion.prob. ratio

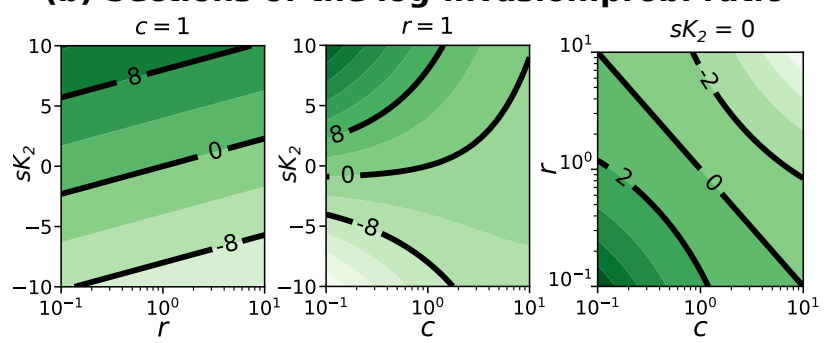

FIG. 2: How the fixation probability depends on the three parameters. Panel (a) shows the $\operatorname{surface} \log \left(p_{1 \mathrm{fix}} / p_{2 \mathrm{fix}}\right)=0$, panel (b) different sections of the fixation probability log-ratio. A darker shade of green represents a larger ratio.

Carrying capacity, inter-generation times, and invasion fitness control the evolutionary success

The evolutionary success of an allele in an environment described by 2 can be quantified by the probability that one individual of a given type is able to invade a population of a second type, i.e the fixation probability. The ratio between the probability of fixation of one individual of the first allele $p_{1 \text { fix }}$ and the fixation of one individual of the second one $p_{2 \text { fix }}$ can be expressed analytically (see Supplementary Materials section S4)

$$
\log \frac{p_{1 \mathrm{fix}}}{p_{2 \mathrm{fix}}}=s K_{2} \frac{r-1}{r-c}+\frac{s K_{2} r(c-1)-(1-r c)(r-c)}{(r-c)^{2}} \log \frac{c}{r} .
$$

Despite the generality of the modeling framework (7), this quantity depends on only three parameters: the intergeneration times ratio $r$, the ratio between the carrying capacities $c$, and the product between the selection coefficient and the size of the second population at carrying capacity $s K_{2}$.

Figure 2a shows the surface at $\log \left(p_{1 \mathrm{fix}} / p_{2 \mathrm{fix}}\right)=0$, which separates the regions of parameters between a more successful first allele, $p_{1 \mathrm{fix}}>p_{2 \mathrm{fix}}$, and the opposite scenario. Note that, differently from the deterministic approximation 1. here, an allele with lower invasion fitness can invade more easily, i.e. for the parameters within the volume below the $s=0$ plane and above the $\log \left(p_{1 \text { fix }} / p_{2 \text { fix }}\right)=0$, surface of the Figure.

In absence of differences in the inter-generation time and carrying capacity (i.e., if $r=c=1$ ), one recovers the Kimura's diffusion limit and the classical result obtained under a constant total population $\operatorname{size} K_{2}[8] \log \left(p_{1 \text { fix }} / p_{2 \text { fix }}\right)=$ $K_{2} s$. 


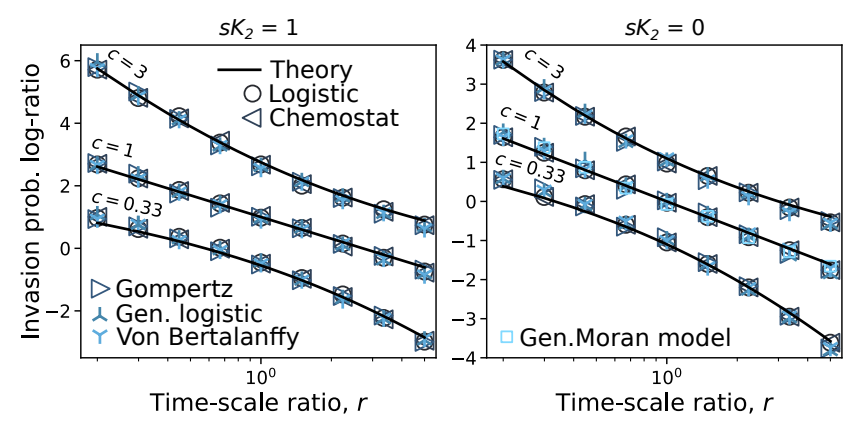

FIG. 3: Collapse of different demographic models on the same fixation probability, given by equation (4), black lines. The simulations consider the competitive Lotka-Volterra, a chemostat model [34], the Gompertz growth dynamics [35, 36], a generalized Lotka-Volterra [37] with exponent $\nu=1 / 2$, the Von Bertalanffy model 38] with $\alpha=2 / 3$ and the Generalized Moran model 31 which is defined only for $s K_{2}=0$ and $c=1$. For more details about the models see Materials and Methods.

The evolutionary outcome is independent of modeling details

One key result of our derivation is that the evolutionary trajectories, described in the diffusion limit by eq. 2, do not depend on the specific modeling choices. More precisely, eq. 2 does not depend on the shape of the functions $\beta(z)$ and $\omega(z)$.

Figure 3 shows numerical simulations of the fixation probability in alternative models, differing for their birth and death rate dependency on the alleles population sizes. The figure shows that once these different models are mapped onto our framework (7), all the invasion probabilities collapse onto the same curves as predicted by equation (4). Details about how the simulations are performed are shown in Supplementary Materials, section S4. The definitions of the simulated models and how to map them in our framework are in Materials and Methods.

Moreover, for $c=1$ and $s=0$ one recovers the fixation probability of [31] and [29].

\section{Fixation probability through mutation}

The expected number of mutations per generation depends on the population size. Since in our case the total population size can vary across population compositions, also the total mutation rate depends on which alleles are present and in which abundance. This dependency induces an additional effect of the varying total population on the most likely evolutionary trajectory.

We consider small, allele-independent, and symmetric mutation probability $U \ll 1$. Given, for example, the population 2 at carrying capacity, $K_{2}$, the birth and death rates of (7) are balanced (the system is in the steady state), $b_{2}=\rho_{2} \beta\left(\omega^{-1}(\phi)\right) K_{2}$. This quantity defines the generation rate. If one multiplies this rate by the per-capita mutation probability, it obtains the the rate at which a mutant of type 1 appears: $U b_{2}$. Therefore, one can compute the probability that the new invader overcomes the resident population in the case it appears because of a mutation: $m_{1 \mathrm{fix}}=U b_{2} p_{1 \mathrm{fix}}$. In particular, it can be easily found that the ratio of this quantity for the two types reads:

$$
\frac{m_{1 \mathrm{fix}}}{m_{2 \mathrm{fix}}}=\frac{c}{r} \frac{p_{1 \mathrm{fix}}}{p_{2 \mathrm{fix}}}
$$

Figure S2 of the Supplementary Materials inspects the analytical behavior of this expression. Also in this case there is 
a large range of parameters for which the population evolves to lower values of the invasion fitness.

\section{Evolution in the chemostat model with metabolic trade-off can revert the direction of evolution}

As an example for the application of the previous results, we consider a population growing in a chemostat on externally provided resources 32 . We consider a birth-death process where the birth-rate depends on the expected availability of resources, while the death rate is a constant factor. In the deterministic limit the equation reduces to

$$
\frac{1}{n_{i}} \frac{d n_{i}}{d t}=\frac{\eta_{i} \alpha_{i}}{\frac{1}{M} \sum_{i} n_{i} \alpha_{i}}-\delta_{i} .
$$

The parameter $\alpha_{i}$ represents the resource intake rate of allele $i, \delta_{i}$ is a death/dilution rate, while $M \eta_{i}$ is the efficiency of resource-to-biomass conversion (equivalent to an inverse yield), and $M \gg 1$ a large parameter. One can then map this model into our general framework (7), see Materials and Methods.

A particularly interesting scenario appears when $\alpha$ is the only parameter that evolves through mutations, while $\delta$ or $\eta$ depend on it. For instance, yield (or, equivalently, efficiency) decreases during selection in experimental evolution [39 41, giving rise to a trade-off between growth rate and yield.

Specifically, we focus on the case $\delta_{i} / \eta_{i}=\alpha_{i}+\alpha_{0}$, where $\alpha_{0} \ll 1$ is a small parameter (in principle of the order of $1 / M$ ). This choice implies that the population size at carrying capacity in a clonal population, i.e. $K_{i}=M \eta_{i} / \delta_{i}=M /\left(\alpha_{i}+\alpha_{0}\right)$, decreases with the intake rate $\alpha$, giving rise to the growth-rate and yield trade-off.

In the deterministic limit, it is easy to see that alleles with higher intake rates (i.e. with higher invasion fitness $\left.\phi_{i}=\alpha_{i} \eta_{i} / \delta_{i}=\alpha_{i} /\left(\alpha_{i}+\alpha_{0}\right)\right)$ always invade populations with lower values of $\alpha$. By using using equations (4) and (5), we show that the presence of a large, but finite, allele-dependent total population size can invert this behavior. Moreover, the outcome of the evolutionary process depends on whether the dependency on $\alpha$ is included in the death rate or in the efficiency. We consider here the former case, while we discuss the latter, which nevertheless leads to similar results, in the Supplementary Materials, section S5.

If the intake conversion from the resource to the biomass is constant, $\eta_{0}$, the death rate per-capita can be expressed as $\delta_{i}=\eta_{0}\left(\alpha_{i}+\alpha_{0}\right)$. The computation of the time-scale ratio leads to $r=c$. In such a case, there is no difference between external invasions, eq. 4 or invasions through mutations, eq. 5. Both the criteria show that the fixation probability is always larger than others for the allele with $\alpha^{*}=\sqrt{\alpha_{0} M / 2}$. The existence of a finite value which maximize the fixation probability implies the existence of an optimal value of $\alpha$ to which the evolutionary trajectories converge.

Figure 4 shows a simulation of this scenario, where $\alpha$ can mutate taking discrete, uniformly spaced, values $\left\{\alpha_{i}\right\}$ with a small mutation probability that guarantees that the regime of periodic selection holds. The evolutionary trajectory, starting both above and below the predicted optimal value converge to it in the long term. Figure $4 \mathrm{~b}$ shows that the expected value of the intake rate $\alpha$ (and therefore the invasion fitness) decreases over time, if the population has initial values of $\alpha$ above the predicted value.

The presence of stochasticity implies that the optimal value is reached on average, with a fluctuating value of $\alpha$ around the optimal value. One can predict the distribution $p(\alpha)$ at equilibrium by assuming that evolution follows a jump process which satisfies the detailed balance: $p\left(\alpha_{i}\right) p\left(\alpha_{i} \rightarrow \alpha_{i+1}\right)=p\left(\alpha_{i+1}\right) p\left(\alpha_{i+1} \rightarrow \alpha_{i}\right)$ (details in Supplementary 

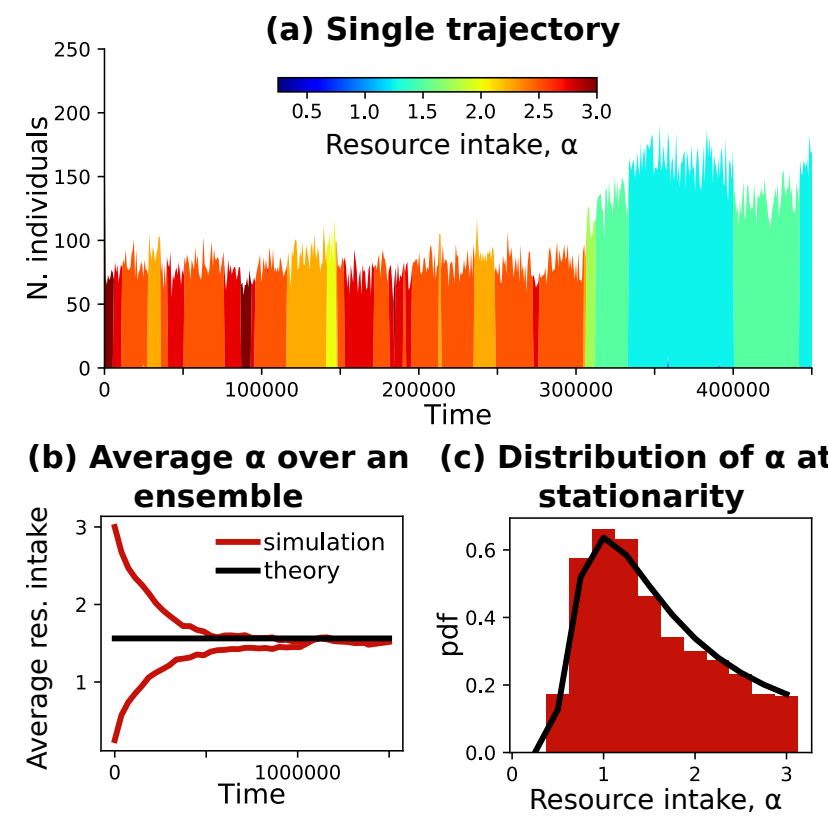

FIG. 4: Evolutionary dynamics of the chemostat with a constant intake rate $\eta_{0}=1$. The resource intake $\alpha$ can mutate to a neighbour $\alpha+\Delta \alpha$ or $\alpha-\Delta \alpha$ with rate $U=2 \cdot 10^{-5}$. It is constrained to be in [0,3]. The other parameters are: $\Delta \alpha=0.25$, $\alpha_{0}=0.01, M=200$. Panel (a) shows the number of individuals colored by their value of $\alpha$ of a Gillespie simulation. There is no clonal interference since, typically, there is no co-existence of sub-populations with different $\alpha$. Panel (b) is the average resource intake over 300 realizations of two ensembles starting from different initial conditions. Panel (c) is the distribution of $\alpha$ at stationarity of the 300 realizations.

Materials, section S5). The prediction for the average and the full distribution is in agreement with the simulation in panels (b) and (c) of Figure 4

Discussion

Here we have studied the evolutionary trajectories of populations in a broad class of models characterizing population self-limitation. It has been introduced a population dynamics framework that encompasses several known models (Logistic, Gompertz, Chemostat, and others). In presence of large, but finite, populations sizes, we have shown that the evolutionary trajectory of these populations depends only on three quantities (the timescale, the invasion fitness, and the carrying capacities), irrespective of models' specifics.

We obtained these results by assuming a time-scale separation between the total population size and the allele frequencies [24, 27, 29, 31]. This assumption allows us to write down an effective equation that describes the time evolution of allele frequencies, which depends only on the values of the three evolutionary relevant quantities. This effective description is akin to Kimura's diffusion, to which it reduces if alleles differ only in their invasion fitness or in the limit of strong selection, where variation in timescale and/or carrying capacities becomes effectively irrelevant.

One especially interesting aspect of this effective equation is the role played by the (finite) population size. In Kimura's diffusion limit, population size influences only the strength of drift. While in that context drift masks the effect of selection (e.g., a deleterious mutation has a non-zero fixation probability), it never alters its direction (i.e., a 
beneficial mutation has always a larger fixation probability than a deleterious one). In our setting, the existence of finite population size might alter the course of evolution: mutants with lower invasion fitness might be more likely to invade than alleles with a higher invasion fitness.

We explicitly show this effect in the chemostat model in the presence of a metabolic tradeoff. In the deterministic limit, larger resource intakes correspond to higher fitness, and therefore evolution drives the population to higher and higher resource intakes. In presence of a large, yet finite, population size, the naive expectation obtained in the deterministic case is not realized. The evolutionary trajectory converges in fact to an optimal value of the intake rate, which we analytically predict. If a clonal population has an initial intake rate larger than the optimal one, evolution will drive the population to decrease the intake rate, and therefore invasion fitness.

Invasion fitness decreases over time because of the presence of finite population size. This might be reminiscent of Muller's ratchet 42 44, but it has a radically different origin. In the case of Muller's ratchet, the decrease in fitness is determined by the fact that mutations only give rise to deleterious mutations, which can get fixed because of the finite population size. The presence of a small fraction of beneficial mutations is enough to balance the effect of deleterious mutations and lead to an overall increase of fitness 45 .

In our case, the mechanism in play is radically different. The presence of variation in the timescale and/or in the carrying capacity creates an effective force driving the population to higher values of the carrying capacity and lower values of the inter-generation times. Lower carrying capacities correspond to a higher level of demographic stochasticity (as shown in eq. S12 of the Supplementary Materials). The intuition is that the evolutionary trajectory drives the population toward lower values of demographic stochasticity. This effect is alike to thermophoresis [46] or to what is observed in Brownian motion in presence of a position-dependent diffusion: an effective force drives the trajectories to lower values of noise [47. In our case, the effective force can counterbalance the presence of a selection coefficient.

This result highlights the relevance of genetic drift in shaping evolutionary trajectories. In our case, drift not only affects the speed of evolution [48, but also its direction. One remarkable aspect is that this effect turns out to be model-independent and determined only by differences in the timescale and carrying capacity.

The three quantities (invasion fitness, carrying capacity, and timescale) that effectively determine the trajectory of an evolving population are not independent traits. Variation of a given trait can influence one, two, or all three of them. For instance, in the example of the chemostat model that we discussed, only one trait, the resource intake, is under selection pressure. But since it influences all three quantities, the result of evolution is far from trivial.

These results go in the direction of building a model-independent eco-evolutionary theory. The extent to which the specific details of ecological interactions influence the evolutionary outcome is a major limitation to the development of a comprehensive general understanding of eco-evolutionary trajectories. Our results present a first step in this direction, as they describe the evolution of a population limited by a single factor. The next step will be to generalize our framework by considering multiple limiting factors, giving rise to the coexistence of different populations [49]. As a further generalization, one can consider different interaction types, going beyond the competition.

To conclude, our work shows that several aspects of the demographic dynamics are details that do not influence the trajectories of an evolving population. Only three life-history traits effectively matters. Their variation is subject to selection in a non-trivial, drift mediated, way, which might lead to a decrease of what is usually identified as fitness over time. 
We consider a population with $A$ alleles. The allele abundances $n_{i}, i=1, \ldots, A$, can increase/decrease of one unit according to birth/death events with rates:

$$
\begin{aligned}
& n_{i} \rightarrow n_{i}+1 \text { w. rate } b_{i}(\{n\})=\frac{n_{i}}{T_{i}} \beta\left(\sum_{j} \frac{n_{j}}{K_{j}} u_{j}\right) \\
& n_{i} \rightarrow n_{i}-1 \text { w. rate } d_{i}(\{n\})= \\
& =\frac{1}{\phi_{i}} \frac{n_{i}}{T_{i}} \beta\left(\sum_{j} \frac{n_{j}}{K_{j}} u_{j}\right) \omega\left(\sum_{j} \frac{n_{j}}{K_{j}} u_{j}\right),
\end{aligned}
$$

where $u_{i}=\omega^{-1}\left(\phi_{i}\right)$. In the deterministic limit, obtained for large population sizes, stochastic fluctuations are neglected and the time derivative of the population abundance $n_{i}$ is given by the difference between birth and death rate, leading to eq. 1 .

The functions $\beta(z)$, and $\omega(z)$ are arbitrary, with the constraints of both being positive for $z>0$, and $\omega$ being monotonic increasing. These conditions guarantee the existence of a globally stable feasible fixed point of the deterministic dynamics (see Supplementary Materials, section S1 for a derivation of a Lyapunov function).

In presence of a clonal population, with only allele 1, the birth and death rates are equal when

$$
\omega\left(\frac{n_{1}}{K_{1}} \omega^{-1}\left(\phi_{1}\right)\right)=\phi_{1},
$$

which occurs when the population abundance $n_{1}$ reaches the carrying capacity $K_{1}$. Above carrying capacity $\left(n_{1}>K_{1}\right)$ the death rate exceeds the birth rate, and below $\left(n_{1}<K_{1}\right)$ births are more frequent than death. The parameter $T$ is related to the inter-generation timescales at carrying capacities: an individual in a clonal population at stationarity $\left(n_{1}=K_{1}\right)$ give birth and dies with rate proportional to $1 / T_{1}$ (where the proportionality constant is given by $\left.\beta\left(\omega^{-1}\left(\phi_{1}\right)\right)\right)$.

The invasion fitness $\phi$ determines the probability that a rare mutant/invader spread in the population in presence of a large resident population at carrying capacity. Let the allele 1 be a rare invader and 2 the type of the resident population. Up to small terms (of the order $1 / K_{1}$ ), the per-capita birth rate of the invader is equal to $\beta\left(u_{2} n_{2} / K_{2}\right) / T_{1}$ while the death rate is $\beta\left(u_{2} n_{2} / K_{2}\right) \omega\left(u_{2} n_{2} / K_{2}\right) /\left(\phi_{1} T_{1}\right)$. If the resident population is at stationarity $\left(n_{2}=K_{2}\right)$, the per-capita birth rate reduces to $1 / T_{1}$ and the death rate to $\phi_{2} /\left(\phi_{1} T_{1}\right)$. The birth rate of the invader exceeds its death rate if its intrinsic fitness $\phi_{1}$ is larger than the intrinsic fitness of the resident population $\phi_{2}$. Moreover, the average growth rate of the invader population, given by the difference of per-capita birth and death rates, equals $\left(\phi_{1}-\phi_{2}\right) /\left(\phi_{1} T_{1}\right)$, as also depicted in Fig. 1p. 
The birth-death model (7) can be approximated, for large values of $K_{i}$, with an Itô stochastic differential equation for each allele

$$
\frac{d n_{i}}{d t}=a_{n_{i}}+\sqrt{2 D_{n_{i}}} \xi_{i}
$$

where $\xi_{i}$ is an uncorrelated Gaussian random variable, $\left\langle\xi_{i}(t)\right\rangle=0,\left\langle\xi_{i}(t) \xi_{j}\left(t^{\prime}\right)\right\rangle=\delta_{i j} \delta\left(t-t^{\prime}\right)$ (see Supplementary Materials, Section S2 for the derivation). The deterministic and diffusion coefficients read:

$$
\begin{aligned}
a_{n_{i}}:= & b_{i}(\{n\})-d_{i}(\{n\})= \\
= & \frac{n_{i}}{T_{i}} \beta\left(\sum_{j} \frac{n_{j}}{K_{j}} u_{j}\right)\left(1-\frac{\omega\left(\sum_{j} \frac{n_{j}}{K_{j}} u_{j}\right)}{\phi_{i}}\right) \\
D_{n_{i}}:= & \frac{b_{i}(\{n\})+d_{i}(\{n\})}{2}= \\
= & \frac{1}{2} \frac{n_{i}}{T_{i}} \beta\left(\sum_{j} \frac{n_{j}}{K_{j}} u_{j}\right)\left(1+\frac{\omega\left(\sum_{j} \frac{n_{j}}{K_{j}} u_{j}\right)}{\phi_{i}}\right),
\end{aligned}
$$

In addition to the limit of large population sizes $\left(K_{i} \gg 1\right)$, under which eq. 10 holds, we consider the limit of small fitness differences $\left(\left|\phi_{i}-\phi_{j}\right| / \phi_{i} \ll 1\right)$. In this case, the dynamics separate in a fast transient followed by slow dynamics (see Supplementary Materials section S3 and Figure S1). The initial trajectory drives the system to a slow manifold of solutions, corresponding to the equilibria of the deterministic dynamics in absence of fitness differences. What follows is a dynamic constrained on the slow manifold.

The "slow" dynamics is determined by the combination of two forces. The genetic drift is pushing the system away from the manifold of solutions. The deterministic part of the dynamics is pushing the system back to the slow manifold. Since these two forces do not act orthogonally to the manifold, they result in a non-trivial combination, which effectively drives the evolution of the system. Imposing the separation of timescales (Supplementary Materials, section S3), one obtains the stochastic differential equation 2, describing the effective stochastic dynamics of the relative abundances $x_{i}=n_{i} / \sum_{j} n_{j}$.

We consider the case of two alleles, while we discuss the general case of $A$ alleles in the Supplementary Materials. In this case, we refer to $x \equiv x_{1}$ as the relative abundance of allele 1 , while the relative abundance of the second allele $x_{2}=1-x$. The effective dynamics for $x$ is given by eq. 2 where

$$
\begin{aligned}
\gamma_{r, c}(x):= & \frac{1-x+x c}{1-x+x r c} \\
\sigma_{r, c}(x):= & \frac{(1-r)(1-x+x c)}{(1-x+c r x)^{2}}(1-2 c-(3+c(c r-4)) x+ \\
& \left.+2(1-c)(1-c r) x^{2}\right) \\
\delta_{r, c}(x):= & \frac{(1-x+c x)^{3}(1-x+r x)}{(1-x+c r x)^{2}} .
\end{aligned}
$$


When the invading allele is rare, eq. 2 can be expanded around $x=0$, from which one obtains

$$
\frac{d x}{d t}=\frac{\gamma_{r, c}(0)}{T_{1}}\left(s-\frac{\sigma_{r, c}(0)}{K_{2}}\right) x+\sqrt{\frac{\delta_{r, c}(0)}{K_{2} T_{1}}} x \eta(t),
$$

The mapping with the Generalized Moran model [31] can be obtained with $\beta(z)=1, \omega(z)=\alpha(1+z), s=0$ and $\chi_{i}=1$. This corresponds to their "quasi-neutrality" case, where $\alpha$, defined as the ratio of death and birth rate is constant for each genotype. The resulting birth-death process is

$$
\begin{aligned}
& n_{i} \rightarrow n_{i}+1 \text { w. rate } \frac{n_{i}}{T_{i}} \\
& n_{i} \rightarrow n_{i}-1 \text { w. rate } \frac{n_{i}}{T_{i}} \alpha\left(1+\sum_{j} \frac{n_{j}}{K}\right),
\end{aligned}
$$


A generalization of the Gompertz growth law [36] for competing species is the following:

$$
\frac{1}{n_{i}} \frac{d n_{i}}{d t}=\alpha_{i} \log \left(\frac{k_{i}}{\sum_{j} \chi_{j} n_{j}}\right)
$$

which recovers the classical Gompertz growth dynamics in the case of one allele and $\chi_{1}=1$. The mapping with the general framework can be done as follows: $\beta(z)=1, \omega(z)=\log (z), T_{i}=1 /\left(\alpha_{i} \log \left(k_{i}\right)\right), \phi_{i}=\log \left(k_{i}\right)$, and $K_{i}=k_{i} / \chi_{i}$.

\section{Generalized Lotka-Volterra}

The generalized logistic dynamics [37 for competing species can be written as follows:

$$
\frac{1}{n_{i}} \frac{d n_{i}}{d t}=\frac{1}{T_{i}}\left(1-\left(\frac{\sum_{j} \chi_{j} n_{j}}{k_{i}}\right)^{\nu}\right)
$$

whose mapping reads: $\beta(z)=1, \omega(z)=z^{\nu}, \phi_{i}=k_{i}^{\nu}$, and $K_{i}=k_{i} / \chi_{i}$. The simulations of figure 3 are obtained for $\nu=1 / 2$.

\section{Von Bertalanffy model}

An extension of the Von Bertalanffy model [38 to competing species reads:

$$
\frac{1}{n_{i}} \frac{d n_{i}}{d t}=p_{i}\left(\sum_{j} \chi_{j} n_{j}\right)^{\alpha-1}-q_{i} .
$$

It is included in the general framework by choosing $\beta(z)=z^{\alpha-1}, \omega(z)=z^{1-\alpha}, T_{i}=1 / p_{i}, \phi_{i}=p_{i} / q_{i}$, and $K_{i}=\left(q_{i} / p_{i}\right)^{1 /(\alpha-1)} / \chi_{i}$. The simulations of figure 3 are obtained for $\alpha=2 / 3$.

\section{Evolution in a chemostat with metabolic tradeoff}

We consider a chemostat with $\delta_{i} / \eta_{i}=\alpha_{i}+\alpha_{0}$, where $\alpha_{0} \ll 1$. The invasion fitness reads $\phi_{i}=\alpha_{i} /\left(\alpha_{i}+\alpha_{0}\right)$ and the carrying capactity $K_{i}=M /\left(\alpha_{i}+\alpha_{0}\right)$. In the case of two alleles we obtain then (at the leading order in $\alpha_{0}$ ) $s K_{2}=M \alpha_{0}\left(\alpha_{1}-\alpha_{2}\right) /\left(\alpha_{1} \alpha_{2}^{2}\right)$ and $c=\alpha_{1} / \alpha_{2}$.

The inter-generation times $T_{i}=1 /\left(\alpha_{i} \eta_{i}\right)$ depend on $\eta_{i}$, but not on $\delta_{i}$. In the main text we consider the case $\delta_{i}=\eta_{0}\left(\alpha_{i}+\alpha_{0}\right)$ and $\eta_{i}=\eta_{0}$, which corresponds to, at the leading order in $\alpha_{0}, r=c=\alpha_{1} / \alpha_{2}$.

Inserting these values of $s K_{2}, r$ and $c$ in eq. 4 one obtains (see also Supplementary Materialssection S4 for the case $r=c)$

$$
\log \frac{p_{1 \mathrm{fix}}}{p_{2 \mathrm{fix}}}=\frac{\left(\alpha_{1}-\alpha_{2}\right)\left(\alpha_{1}+\alpha_{2}\right)\left(\alpha_{0} M-2 \alpha_{1} \alpha_{2}\right)}{2 \alpha_{1}^{2} \alpha_{2}^{2}} .
$$

275 Without loss of generality, let us assume that $\alpha_{1}>\alpha_{2}$. The first allele is advantaged $\left(\log \frac{p_{1 \mathrm{fix}}}{p_{2 \mathrm{fix}}}>1\right)$ only if $\alpha_{0} M-2 \alpha_{1} \alpha_{2}>0$. The allele 1 is advantaged over allele 2 if $\alpha_{2}<\alpha_{1}<M \alpha_{0} /\left(2 \alpha_{2}\right)$. Therefore if an allele $i$ has $\alpha_{i}$ 
[1] M. Kimura, Genetics 47, 713 (1962).

[2] W. J. Ewens, Theoretical population biology 3, 87 (1972).

[3] J. F. C. Kingman, Stochastic processes and their applications 13, 235 (1982).

[4] R. R. Hudson, M. Kreitman, and M. Aguadé, Genetics 116, 153 (1987).

[5] F. Tajima, Genetics 123, 585 (1989).

[6] S. A. Sawyer and D. L. Hartl, Genetics 132, 1161 (1992).

[7] Z. Yang and J. P. Bielawski, Trends in ecology \& evolution 15, 496 (2000).

[8] J. H. Gillespie, Population genetics: a concise guide (JHU Press, 2004).

[9] W. J. Ewens, Mathematical population genetics 1: theoretical introduction, vol. 27 (Springer Science \& Business Media, 2012).

[10] S. Wright, Genetics 16, 97 (1931).

[11] R. A. Fisher, The genetical theory of natural selection ( , 1958).

[12] P. A. P. Moran, in Mathematical proceedings of the cambridge philosophical society (Cambridge University Press, 1958), vol. 54 , pp. $60-71$.

[13] S. Karlin and J. McGregor, Proceedings of the National Academy of Sciences of the United States of America 51, 598 (1964).

[14] C. Cannings, Advances in Applied Probability pp. 260-290 (1974).

[15] J. E. Barrick and R. E. Lenski, Nature Reviews Genetics 14, 827 (2013).

[16] A. Sanchez and J. Gore, PLoS Biol 11, e1001547 (2013).

[17] A. R. Rogers and H. Harpending, Molecular biology and evolution 9, 552 (1992).

[18] O. Hallatschek, P. Hersen, S. Ramanathan, and D. R. Nelson, Proceedings of the National Academy of Sciences 104, 19926 (2007).

[19] O. J. Burton, B. L. Phillips, and J. M. Travis, Ecology letters 13, 1210 (2010).

[20] W. J. Ewens, Heredity 22, 438 (1967).

[21] S. P. Otto and M. C. Whitlock, Genetics 146, 723 (1997).

[22] L. M. Wahl and P. J. Gerrish, Evolution 55, 2606 (2001).

[23] Z. Patwa and L. M. Wahl, Journal of The Royal Society Interface 5, 1279 (2008).

[24] T. L. Parsons and C. Quince, Theoretical population biology 72, 121 (2007).

[25] T. L. Parsons and C. Quince, Theoretical population biology 72, 468 (2007).

[26] Y. T. Lin, H. Kim, and C. R. Doering, Journal of Statistical Physics 148, 647 (2012).

[27] G. W. Constable and A. J. McKane, Physical review letters 114, 038101 (2015).

[28] T. Chotibut and D. R. Nelson, Physical Review E 92, 022718 (2015).

[29] T. Chotibut and D. R. Nelson, Journal of Statistical Physics 167, 777 (2017).

[30] P. Czuppon and A. Traulsen, Journal of mathematical biology 77, 1233 (2018).

[31] T. L. Parsons, C. Quince, and J. B. Plotkin, Genetics 185, 1345 (2010).

[32] H. L. Smith and P. Waltman, The theory of the chemostat: dynamics of microbial competition, vol. 13 (Cambridge university press, 1995).

[33] T. L. Parsons and T. Rogers, Journal of Physics A: Mathematical and Theoretical 50, 415601 (2017). 
[34] A. Posfai, T. Taillefumier, and N. S. Wingreen, Physical review letters 118, 028103 (2017).

[35] B. Gompertz, Philosophical transactions of the Royal Society of London pp. 513-583 (1825).

[36] C. P. Winsor, Proceedings of the National Academy of Sciences of the United States of America 18, 1 (1932).

[37] F. Richards, Journal of experimental Botany 10, 290 (1959).

[38] L. Von Bertalanffy, The quarterly review of biology 32, 217 (1957).

[39] F. Vasi, M. Travisano, and R. E. Lenski, The american naturalist 144, 432 (1994).

[40] M. Novak, T. Pfeiffer, R. E. Lenski, U. Sauer, and S. Bonhoeffer, The American Naturalist 168, 242 (2006).

[41] J.-N. Jasmin, M. M. Dillon, and C. Zeyl, Proceedings of the Royal Society B: Biological Sciences 279, 4382 (2012).

[42] H. J. Muller, Mutation Research/Fundamental and Molecular Mechanisms of Mutagenesis 1, 2 (1964).

[43] W. Gabriel, M. Lynch, and R. Bürger, Evolution 47, 1744 (1993).

[44] R. A. Neher and B. I. Shraiman, Genetics 191, 1283 (2012).

[45] S. Goyal, D. J. Balick, E. R. Jerison, R. A. Neher, B. I. Shraiman, and M. M. Desai, Genetics 191, 1309 (2012).

[46] R. Piazza, Soft Matter 4, 1740 (2008).

[47] S. Chapman, Proceedings of the Royal Society of London. Series A, Containing Papers of a Mathematical and Physical Character 119, 34 (1928).

[48] M. M. Desai and D. S. Fisher, Genetics 176, 1759 (2007).

[49] B. H. Good, S. Martis, and O. Hallatschek, Proceedings of the National Academy of Sciences 115, E10407 (2018). 